\title{
Cold Atoms Go Topological
}

\author{
A lattice of highly excited atoms can exhibit a topological phase, a new \\ theoretical study shows.
}

By Sophia Chen

$\mathrm{T}$

o distinguish a loop of yarn from a single, tangled strand, you have to follow its entire length until you either find an end or arrive back at the beginning. In a similar way, some materials can adopt configurations that cannot be identified from local measurements; they must be examined as a whole. Theorists predict a myriad of applications for these "topologically ordered states," as topological order has been linked to superconductivity and offers desirable properties for quantum computing. Now, Ruben Verresen of Harvard University and colleagues demonstrate theoretically that such a topological phase can be exhibited by cold atoms on an engineered lattice [1]. Realizing their proposal experimentally would constitute a rare observation of topological phases in a material.

The researchers propose to create a phase with so-called $Z_{2}$ topological order in a 2D array of cold atoms. Each atom would form a vertex in a "ruby" lattice consisting of triangles, rectangles, and hexagons. The team would excite some of the atoms into an extremely high quantum state known as a Rydberg state. Adjacent atoms cannot be excited into this state simultaneously, as one Rydberg atom shifts its neighbor's

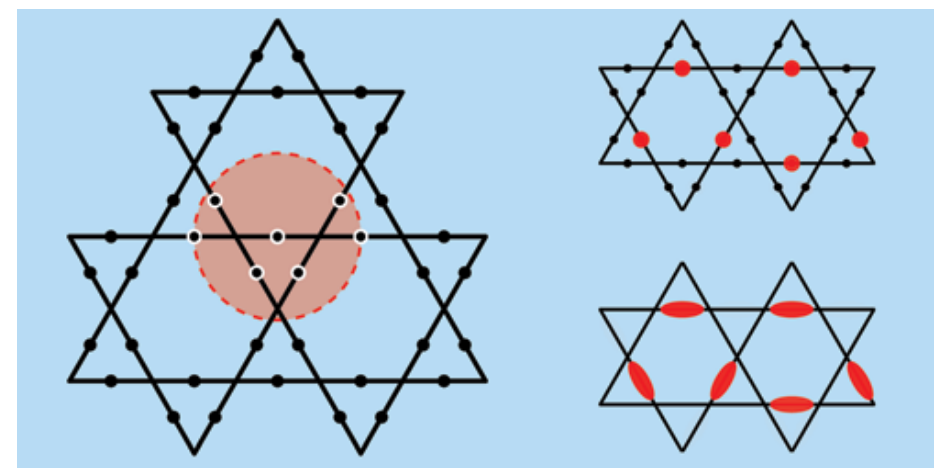

Credit: R. Verresen et al. [1] 